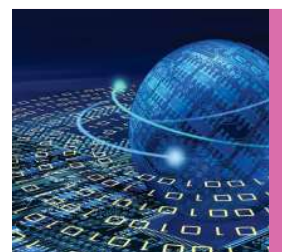

\title{
A Survey on Emotion Detection from Text in Social Media Platforms
}

\author{
M. Usman Ashraf', Moeed Rehman ${ }^{1}$, Qasim Zahid ${ }^{1}$, Mustahsan Hammad Naqvi ${ }^{1}$, and Iqra Ilyas ${ }^{2}$ \\ ${ }^{1}$ University of Management and Technology, Sialkot, Pakistan \\ ${ }^{2} \mathrm{GC}$ Women University Sialkot, Pakistan \\ Email: usman.ashraf@skt.umt.edu.pk
}

\begin{abstract}
This paper provides an overview of the evolving field of emotion detection and identifies the current generation of methods of emotion detection from social media platforms as well as the challenges. The challenges in the field of current emotion detection are discussed in detail and potential alternatives are proposed to enhance the ability to detect emotions in real-life systems that emphasize interactions between humans and computers as well as advertisements, recommendation systems, and medical fields such as computer-based therapy. These solutions include the extraction of semantic analysis keywords, and ontology design with the evaluation of emotions. There are multiple models and classifications of emotions such as Ekman's model (Happy, Anger, Sad, Disgust, Fear, Surprise), and Plutchik's model (anger-fear, surprise-anticipation, joy-sadness, joy-sadness). Further, a systematic review of publications on textual emotions detection from social media platforms, state-of-the-art methods, and existing challenges presented. Finally, we conclude with some recommendations based on critical analysis of existing techniques and determine future research directions presented at last.
\end{abstract}

KEYWORDS: Emotion Detection, Social Media, Natural Language Processing.

\section{INTRODUCTION}

In recent years, the text has been the main form of communication on social media platforms such as Facebook, Twitter, Tumblr, Reddit as well as many other countless platforms. Emotion Detection from these platforms can be used to excel in fields such as human-machine interactions, recommendation systems, advertisements, and computer-based therapy $[1,2]$. To analyze such data from these platforms and detect emotions we need several types of techniques such as Semantic Neural Networks, NLP (Natural Language Processing), DL (Deep Learning), Parallel computing [28], EVM [34], and other techniques discussed in [3, $4,6]$. Compared to the emotion detection research in other fields, emotion detection from text is still not mature enough and needs further enhancements to be assembled in real-life applications $[8,11]$.

The enhancements include a better understanding of newly developed terminologies, the integration of psychological theories behind the texts to infer emotions, the use of contextual information, the development of more sophisticated methods of emotion detection that enable more emotional and inference categories [14, 18, 23]. Emotions are divided into classifications based on different researches done. The most common classification is Ekman's model (Happy, Anger, Sad, Disgust, Fear, Surprise). Later on, Paul Ekman improved his work and implemented pride, shame, embarrassment, and excitement into his previous list [26]. Plutchik's model (anger-fear, surprise-anticipation, joy-sadness, joy-sadness) compares emotions based on their polar-opposite emotions $[27,40]$. There are other emotion classification models but the emotion detection models we studied only used these. The challenges discussed in the paper revolve around the detection of emotions and how can they be improved. These challenges range from Accuracy, Efficiency, Emotion Classifications, Writing Styles, Fuzzy Boundaries, Grammatical 
and Syntactical Structures [32], Emotion Variety, and the existence of Multiple Emotions in text. The main purpose of this paper is to analyze different new models proposed from 2018 to 2020 and describe their challenges as well as make comparative analysis based on their characteristics, technologies used, and their limitations in table 1. Based on our research and discussion, we then recommend which model would be the best for real-world applications.

In this paper, we discuss objectives, challenges, and existing models. These Challenges are discussed in Section II, Section III talks about the Related Work we studied for this review paper, Section IV focuses on Discussion and Recommendations, and lastly, in Section V we have our Conclusion.

\section{E M O T I O N D E T E C T I O N CHALLENGES}

\subsection{Accurate Emotion Detection}

Emotion accuracy is one of the key challenges facing the models of Emotion Detection $[1,5,7,12,24]$. Accuracy is one of the key variables deciding the model's success rate. It is a complex challenge that needs to be tackled in almost every model introduced to get precise emotions.

\subsection{Accurate Sentiment Analysis}

Sentiment accuracy is just as critical a challenge as emotion detection in the identification of emotions $[3,4,5,29]$. The sentiment is a significant factor that decides whether the emotion was positive or negative and even the intensity of the polar opposite emotions could make a huge difference in emotion identification so the models that are presented need to be accurate in sentiment analysis as well as emotion detection accuracy.

\subsection{Emotion Classification}

Emotion classification is also one of the main challenges which are faced in the Emotion Detection models [3, 5, 6, 7, 9 18]. The words need to be assigned a classification based on the emotion they represent, if there is no classification of the text data then the detection cannot be performed correctly and the emotions cannot be assigned to the text's underlying meaning. The models need to take into consideration that the datasets they use need to be rich.

\subsection{Casual Writing \\ Casual writing is one of the key}

challenges $[6,11]$. The use of a hashtag in posts is pretty common but what the model might detect may be outside the real sense of the post. Words such as slangs and abbreviations also count in casual style writing which needs to be addressed to become properly understandable for the system to detect the emotions from the text. Another problem that appears in the challenge is that words, especially in informal writing, keep on expanding and more and more words keep on adding into the dictionary as time passes.

\subsection{Fuzzy Boundaries}

The use of emoticons is as widespread on social media platforms like the use of text and it keeps on growing, so emoticons must also be taken into consideration in challenges for a textbased emotion detection model $[3,7,10,11]$. In the raw text, emoticons can often show emotions that were not present in the text alone. Emoticons themselves may present another challenge that is that the emoticons used could be ambiguous or irrelevant to the text. Just like casual writing, emoticons get updated as time passes so the model needs to take into consideration of new emoticons that get introduced as well.

\subsection{Efficiency}

Efficiency is an important challenge that needs to be tackled as well $[1-4,7,13,18,22]$. In real-world applications, a model that takes too long to detect emotions and perform sentiment analysis, as well as language processing, can be considered almost obsolete. When it comes to real-world applications, efficiency is as relevant as precision and accuracy.

\subsection{Sentence Structure}

Detecting emotions from ambiguous or incorrect sentence structures can also be a challenge in the emotion detection models which needs to be addressed $[4,11]$. The model would not be able to identify the exact emotion accurately from the text if any sentences are using incorrect sentence structure.

\subsection{Grammatical Mistakes}

Grammar mistakes can also be a challenge in emotion detection models which also needs to be addressed [13]. Some sentences may contain imperfect grammar that may not convey a proper meaning so that the model could not be detecting it correctly. If perfect grammar is used in a sentence, the emotions of the sentence could easily be detected by the model [34-40]. 


\subsection{Emotion Variety}

Human emotions are very complex and numerous. Such emotions are categorized into various classifications such as Ekman's classification, Plutchik's classification, and various other classifications that divide emotions into many more categories. The model for emotion detection should use a large number of emotions that should be identified accurately but it can be a difficult task $[2-5,7,9,11-17,19$, 21-25].

\subsection{Multiple Emotions}

One of the biggest problems in detecting emotions is if the text contains multiple emotions. One emotion in the text could be a complete contrast to another emotion detected in the same text which could lead to problematic and unreliable emotion detection which would later lead to lower accuracy $[8,41]$. This is a challenge that needs to be addressed because a lot of text, especially on social media platforms, contains varying amounts of emotions.

\section{EMOTION DETECTION STATE- OF-THE-ART METHODS}

Batbaatar. E., Li. M., \& Ryu. K. H., [1] elaborated on Neural Network for Emotion Detection Semantic-Emotion From Text. The objective of the proposed research is to come up with a new model that can detect emotion much more accurately than the models presented before. To achieve the above objective, the authors presented a new model called SENN (Semantic-Emotion Neural Network) to get significantly superior emotion recognition from text. By adopting pre-trained word representations, the suggested model utilizes both semantic/syntactic and emotional information. SENN has used two main subnetwork, the first one uses BiLSTM (Bidirectional Long-Short Term Memory) to focus on the semantic relationship and to get contextual information, the second sub-network uses a CNN (Convolutional Neural Network) to focus on the emotional relationship between words from the text and to get emotional features. In contrast, the proposed model SENN had some small challenges, which required it to be addressed like performance using larger emotion word embedding. These challenges are still new for this research model which opens new opportunities for researchers and development communities.

Jia. Z., [2] explained Deep Learning and NLP(Natural Language Processing) in Artificial
Intelligence. The objectives of the proposed study are to make it go with greater accuracy than the previously produced models, to train the model more quickly, and to generalize the model as well. To achieve the above objectives, the authors have presented a new model called BiLSTM-LM (Bi-directional Long Short-Term Memory Language Model) in which the text series will be divided into six separate emotional categories. Word embedding techniques have also been used to accurately encode the lexicons and the text will be shown up as the feature word representation and feature character representation. The drawbacks the authors were facing were: length dictionary is the length of each dimension of the matrix, and the one-hot vector corresponding to each word is the vector of 1 X10,000. Some of the challenges mentioned in the study are still new and can help the upcoming researchers to work on.

Tashtoush. Y. M., \& Orabi. D. A. A. A., [3] described on Tweets Emotion Prediction by Using Fuzzy Logic System. The objective of the proposed research is to establish sentiment analysis systems that extrapolate the emotions a tweet (text) holds in its words and emojis by using the multi-labeling fuzzy logic approach for the classification logic and to see which is more reliable for emotion detection, text or emojis. To achieve this objective, the authors presented two new Fuzzy Classification Systems, the first one processed the text and is named TFCL (Textual Classification by Fuzzy Logic), where the second one processed the emojis associated with the tweet's text and is named ECFL (Emojis Classification by Fuzzy Logic). In contrast, the proposed Fuzzy Classification Systems had some challenges which needed to be addressed like both the classification systems weren't united, the main number of emotions needed to be increased, and increased reliability was still to be achieved. These challenges are still new in research directions and leave opportunities for researchers and development communities.

Xiangsheng. L., Jianhui. P., Biyun. M \& Yanghui. R., [4] discussed Machine Learning and NLP (Natural Language Processing) in Artificial Intelligence. The objectives of the proposed study are to make the neural network's hidden layer approximate the interface of BTM (Biterm Topic Model), also to enhance the performance of the supervised learning. To achieve the above objectives, the authors have proposed a new model which is called HNN 
(Hybrid Neural Networks) to resolve the emotion detection issues to eliminate failure problems. HNN contains three steps: The number of hidden neurons is selected first, then pre-training based on LSM (Latent Semantic Machine) and fine-tuning based on the hybrid model at the end. What models the authors have chosen to set the parameters of all the neural networks consistently are SWAT, ET, ETM, MSTM, and ATM. The drawback the authors were facing was the instability problem which was becoming more extreme than the neural networks with one hidden layer, which is normal for the extension of neural networks. Some of the problems identified in the study are still new and may encourage future researchers to work on them.

Hassan. M., Alam. M. S. B., \& Ahsan. T., [5] explored Emotion Detection from Text Using Skip-Thought Vectors. The objective of the proposed research is to present emotion detection from text using a newly proposed deep learning model called the Skip-Thought model by using a model which embeds the pre-trained sentences for classifying emotions. To achieve the above-described objective, the authors proposed a new framework that embeds the Skip-Thought model's trained encoder which takes advantage of the pre-trained model and pre-trained word vectors along with the lookup table layer. In contrast with the objectives, the proposed framework in this research paper needs some improvements which need to be addressed like the investigation of how other word vectors such as word2vec and Glove can be implemented in this emotion detection logic using Skip-Thought Vectors as well as usage of this framework on large-scale pseudo-labeled datasets for better and more accurate results. These challenges are still new in the research field and open opportunities for researchers and development communities.

Maryam. H., Elke. R., \& Emmanuel. A., [6] illustrated on Machine Learning in Artificial Intelligence. The objective of the proposed study is to detect the emotions in social networks through classifying the text messages into many different classes of emotion. To achieve these goals, the authors have tackled the challenges like casual style of microblogs, semantic complexity of text messages, fuzzy boundaries of emotions, the difficulty of emotion annotation, multiple topics, and emotional states, and inconsistent annotators. To achieve the above objectives, the authors have presented a dimensional model of effect that has been utilized for the definition of emotion classes, plus a soft classification approach has also been proposed for the measurement of the chances of assigning a message to every emotion class. The authors have developed and evaluated a supervised learning system to classify the emotion from the text message streams automatically. The drawback of this basic emotion model is, among scholar, there is no consensus on what human emotions should be included in the basic collection of emotions.

Shah. F. M., Reyadh. A. S., Shaafi. A. I., Ahmed. S., \& Sithil. F. T. [7] expounded on Emotion Detection from Tweets using AIT-2018 Dataset. The object of the proposed research paper is to propose a new model for emotion detection using the AIT-2018 dataset that has better accuracy and performance than previously proposed models such as WordNetAffect. To achieve the above-described objective, the authors proposed a new model consisting of EmoSenticNet and WordNetAffect with supervised classifiers for detecting emotions using a gold dataset of Twitter called AIT-2018. In contrast, the proposed model still had some limitations which need to be addressed such as a small sample dataset was used and there is still some language ambiguity because texts containing multiple emotions at the same time were not used. These challenges are still new in this research direction and present opportunities for researchers and development communities to improve on the work done.

Jiangping. H., Chunli. X., Shuwei. Y., Desen. Y., \& Xiaorui. H., [8] elaborated on Deep Learning and NLP (Natural Language Processing) in Artificial Intelligence. The objectives of the proposed study is to allow a computer to understand what a human communicates in our daily lives, to use language to communicate not only with semantic knowledge, but also with emotions, and to determine the strength of the level of emotions. To achieve the above objectives, the authors have presented a model named character-aware convolutional recurrent networks with selfattention for emotion detection on usergenerated content. This model contains three parts: The character-level convolutional layer is designed to learn the representation of words based on pre-trained word embedding, the sequential information used by forwarding and backward repetitive neural networks is learned by recurrent neural networks, and the self- 
attention module is used to extract various emotions into multiple vector representations. The drawback of this study is it cannot detect multiple emotions from a single text or tweet, plus if there is an emoji in a sentence the would this model be detecting the emotions from it? And that could be its another drawback. Zhang. X., Li. W., Ying. H., Li. F., Tang. S., \& Lu. S., [9] explained on Emotion Detection in Online Social Networks: A Multi-label Learning Approach. The objective of the proposed research is to address multiple-level emotion detection in online social networks from a user-level view and formulate a new multi-label-based learning emotion detection system. To achieve the described objective, the authors discovered social correlation and emotion label correlation as well as temporal correlation in online social networks. They also proposed a factor graph model to incorporate the correlations mentioned above. Then they introduced a multi-label learning algorithm to address the problem. In contrast, the proposed system had some drawbacks which need to be addressed like a small-scale dataset was used because of the lack of manpower to produce a bigger dataset. These challenges are still new in research directions and open opportunities for researchers and development communities.

Forugh. M., Hooman. T., [10] described Artificial Intelligence. The objectives of the proposed study are to recognize and classify human emotions based on the writings using similarity techniques and using VSM and STASIS methods. To achieve the above objectives, the authors have proposed two methods in the study to detect emotions, the first method is semantically based in which they used the STASIS method to direct the level of similarities between multiple short-sized texts through WordNet which shows the ontological relation of the words. The second method uses VSM (Vector similarity Measure) which uses the feature vector and cosine similarities for detecting the emotions. One of the drawbacks of this analysis is that the STASIS approach determines the shortest path without paying attention to the original meaning of the terms, which has been improved in this paper but is still a drawback that can cause issues while detecting emotions. Some of the challenges mentioned in the study are still new and can help the upcoming researchers to work on.

Sailunaz. K., \& Alhajj. R., [11] discussed Emotion and sentiment analysis from the Twitter text. The purpose of the research paper is to analyze and detect emotions and sentiment from the Twitter text for the usage of creating recommendation systems. To achieve the above objective, the authors used a dataset to find emotion and sentiment from text tweets and even their responses to gather the influence scores based on a few criteria. Then this information was used to build a framework for the recommendation system. The work done in this paper includes tweets as well as their responses, the authors even introduced agreement score, emotion score, and sentiment score of the replies to collect the influence score, then based on that they developed a recommendation system. On the other hand, the proposed research had some drawbacks which need to be discussed like only simple text and proper words without emojis, abbreviations, and shorthand words were used for the research. This leaves the door open for new research and development communities to add improvements to the proposed research.

Nazia. A. S., Minhas. R., Nasif. A., Raihan., Afsana. I., \& Tanzil. R., [12] explored NLP (Natural Language Processing) in Artificial Intelligence. The objectives of the proposed study is to optimize the results, to collect the tweets data and then mark them up manually, to train the dataset and preprocess the data using unigram with a POS tag, and to extract features. To achieve the above objectives, the authors have used the already existing models i.e. unigram and unigram with POS tag which is for feature extraction, and also used Naive Bayes for the analysis of the emotions, Naive Bayes used to perform better text classification. NLTK has also been used which is a library and built-in program for the statistical and symbolic NLP which is written for Python. These all have been used by the authors to maximize the effects of text emotion analysis. One of the drawbacks they faced is, they have improved the emotion analysis in the text by a few percent but still could not find the exact/correct emotion according to the text, which can be done by finding more than two unique emotions if they use the huge training data.

Ghanbari-Adivi. F., \& Mosleh. M., [13] proposed Text emotion detection in social networks using a novel ensemble classifier based on Parzen Tree Estimator (TPE). The purpose of the research paper is to create a novel ensemble classifier that is efficient and consists of 1500 Multilayer Perceptron, Decision Tree 
basic classifiers, and k-Nearest Neighbour. To achieve the objective, the authors did exactly what they planned out to do and created an emotion detection system that was efficient as well as uses a novel ensemble classifier based on Decision Trees, k-Nearest Neighbor, and Multilayer Perceptron, as well as Parzen Estimator, is used to fine-tune the classifiers in addition to Doc2Vector algorithm. In contrast to the objectives achieved, the proposed research had some drawbacks which need to be discussed like time complexity was very high than other classifiers other than $\mathrm{CNN}$ which needs to be reduced. These drawbacks however open the door for future improvements from the research and development communities.

Hao. F., Yue. Z., Yafeng. R., \& Donghong. J., [14] illustrated on NLP (Natural Language Processing) in Artificial Intelligence. The objectives of the proposed study is to latent the topic information without external resources, to effectively leverage it into a capsule-based classifier for the multiple emotions predictions, also to enhance emotion detection performance. To achieve the above objectives, the authors have proposed a topicenhanced capsule network containing a variational autoencoder and a capsule module for the multilabel emotions detection task. The latent topic information of the text can be learned by the variational autoencoder, and all the rich features for the corresponding emotions can be captured by the capsule module. The drawbacks were two types of incorrect predictions. The first one was a deep semantic understanding of the text, and the second type of error comes from the topic modeling. Some of the challenges mentioned in the study are still new and can help the upcoming researchers to work on.

Canales. L., Daelemans. W., Boldrini. E., \& Martínez-Barco. P., [15] expounded on EmoLabel: Semi-Automatic Methodology for Emotion Annotation of Social Media Text. The objective of the proposed study is to propose a semi-automatic methodology that is based on pre-annotation consisting of an automatic preannotation process to illustrate or pre-annotate the unlabeled English phrases and a manual refinement process where human illustrators decide the dominant emotion. To achieve the above-described objectives, the authors proposed a solution called Emo-Label which is a semi-automatic two-staged technique where an automatic process to pre-annotate unlabeled phrases with a reduced number of categories of emotions, and a manual process of purification in which human illustrators decide the dominant emotion between the pre-defined collection of possibilities. On the other hand, the proposed solution had some drawbacks which need to be addressed. These challenges are still new and need a lot of improvement to overcome but they do leave the door open for future researchers and development opportunities.

Erfian. J., \& Rizal. R., [16] elaborated on NLP (Natural Language Processing) in Artificial Intelligence. The objective of the proposed study is to make the sentences or text classifications into four different emotional classes, named as anger, joy, fear, and sadness which are from six Ekman's emotions. To achieve the above objective, the authors have presented a classifier method that was optimized by particle swarm optimization and this classifier is called Naïve Bayes classifiers. The NBC (Naive Bayes Classifiers) method is an algorithm that is often used in the classification of text documents. This algorithm is used because of its accuracy and simplicity in the text's classification. To represent a solution candidate from a set of cooperated particles, Particle Swarm Optimization was used. The drawbacks the authors were facing is how a word or a sentence can be used to recognize a person's emotions while the person is writing it. These challenges are still new for this research model which opens new opportunities for researchers and development communities.

Kratzwald. B., Ilic. S., Kraus. M., Feuerriegel. S., \& Prendinger. H., [17] explained on Deep learning for affective computing: Textbased emotion recognition in decision support. The proposed research paper presents a model that focuses on accuracy as well as complexity, the ambiguity of language, and performance in particular. To achieve this target, the authors made improvements in recurrent neural networks with regards to bidirectional processing. The authors also proposed a new tailored form of affective computing called sent2affect, which is pre-trained for various tasks while the output layer focuses on emotion detection. The drawbacks that the authors had to face were that the model used a very limited variety of emotions which needs to be addressed. These challenges in the field are quite new which opens new opportunities for researchers and development communities alike. 
Robert. D., \& Dmitrij. S., [18] described ML (Machine Learning) in Artificial Intelligence. The objective of the proposed study is to refer the text document to one or more predefined classes based on its captivating, and this task can be performed by using some Machine Learning approaches. To achieve the above objective, the authors have implemented a Neural network that is based on machine learning and is widely used in NLP (Natural Language Processing) issues. They have also implemented a model named Feature Extraction that plays a key role in text classification as it directly affects the accuracy of the classification. The drawbacks the authors were facing is: this method can generate features that probably have a large number of dimensions if the text corpus is huge, which could probably increase the likelihood of overfitting the classification model. These challenges are still new for this research model which opens new opportunities for researchers and development communities.

Bandhakavi. A., Wiratunga. N., Massie. S., \& Padmanabhan. D., [19] discussed Lexicon generation for emotion detection from text. The proposed research paper presents a new model which focuses input corpus of emotion-lexicons from documents in addition to emotions labels. The achieve such results, the authors divided their model into three main parts, the first part focuses on Unigram Mixture Model (UMM) to learn a word-emotion association from the documents, the second part focuses on the empirical quality of the emotion language, the third part focuses on the quality of the lexicons. The drawbacks authors faced in their research is that they did not use a multi-word lexicon in their model which needs to be addressed. Although this challenge is quite new, this opens a door for future researchers and development communities.

Devamanyu. H., Sruthi. G., Soujanya. P., \& Roger. Z., [20] explored Neuroscience, ML (Machine learning), NLP (Natural Language Processing). The objective of the proposed study is to make an attention mechanism capable of assigning the modalities with suitable ratings. Feature-level fusion between early, late, or hybrid fusion has been the most challenging. To achieve the above objective, the authors have proposed a self-attention mechanism based new feature-level fusion method. The authors conducted a noise stability test in which a multidimensional attention module was found to perform best for fusion, and compared it with conventional methods of fusion such as concatenation, outer product, etc. The drawback the authors were facing is: system degradation is unwanted with the increase in noise in a specific channel and this challenge is still new for this research model which opens new opportunities for researchers.

Haryadi. D., \& Kusuma. G. P., [21] illustrated in detail Emotion Detection in Text using Nested Long Short-Term Memory. The proposed study in this research paper focuses on a model that uses machine learning techniques on Ekman's emotions to get more accuracy. To achieve this target the authors trained the model using Long Short-Term and Nested Long ShortTerm Memory. They trained each model with trained and tested data. The drawback that needs to be addressed is that the authors faced was that their results were not significantly different from other models. These drawbacks are still new and leave the door open for future research and development communities.

Faisal. M. S., Farzad. A., Sajid. K. S. J., Sifat. A., Samir. S., Rimon. S., \& Hasanul. K., [22] expounded on DL (Deep Learning) in Artificial Intelligence. The objective of the proposed study is to detect depression early by analyzing the posts of Reddit users. To achieve the above objective, the authors have proposed a hybrid model that can detect depression by analyzing user's textual posts. The authors have used the feature extraction model, text classification model and also have trained the Deep Learning algorithms using the training data and then the performance was evaluated by them on the test data of the dataset of Reddit. The drawback the authors were facing is that though the users are correctly classified, it takes too long to detect them as depressed and this opens an opportunity for the upcoming researchers to work on.

Fathy. S., El-Haggar. N., \& Haggag. M. H., [23] elaborated on a hybrid model for emotion detection from text. In the proposed paper, the authors presented a new hybrid model to detect emotions from the text which dependant on semantic keywords similarities. To achieve the desired results, the authors extracted the input sentences and matched them with their ontological emotions. Then the extracted sentences are put into the algorithm and checked for the highest score. On the other hand, the proposed model faced some drawbacks which need to be addressed. In addition to that, these drawbacks leave the 
opportunity for the research and development communities to improve the model.

Dibyendu. S., Uttam. K. R., \& Rohini. B., [24] described on NLP (Natural Language Processing) in Artificial Intelligence. The objective of the proposed study is to detect emotions from the emotion database by searching keywords. To achieve the abovementioned objective, the authors have proposed an emotion extraction method that broadly consists of pre-processing text, extraction of keywords from phrases, and examination of keywords to find the emotional affinity of the keyword. By searching emotional words from a pre-defined emotional keyword database that analyzes emotion words and phrasal verbs, they have proposed an effective emotion detection technique, which also considers negation words and exhibits better output than recent approaches. The drawback the authors were facing is that there is still no exhaustive list of emotional keywords in the emotion database, and more tools are still being analyzed by the authors to enhance accuracy.

Calefato. F., Lanubile. F., \& Novielli. N., [25] discussed on Emotxt: a toolkit for emotion recognition from text. The authors in this research paper proposed an open-source toolkit called EmoTxt for emotion recognition in the text as well as the functionality of custom emotion classification of models. To achieve this desired result, the authors trained and tested gold standard questionnaires and their answers from online websites. The drawbacks the authors faced and plan to resolve in the future is that the model needs to validate other gold standards from varying sources which need to be addressed. On the other hand, these drawbacks are fairly new but it leaves the opportunities for future research and development communities to introduce their improvements.

\section{DISCUSSION \& RECOMMENDATIONS 4.1. Discussion}

This review paper is about the models used for the detection of emotions from the text available on different social media platforms, as we have discussed in Section I. This complete analysis of varying models relies mostly on Natural Language Processing, through which the models introduced in the proposed studies would detect emotions based on different classifications of emotions introduced in human psychological studies. As discussed in Section II, emotion detection from the text on social media requires many factors to work together to function properly and to be able to be used in practical real-world applications. The factors mentioned above need to be resolved in newly proposed models for much more accurate and

Table 1: Emotion detection challenges with a short description

\begin{tabular}{|l|l|l|}
\hline Sr. No. & Challenges & Description \\
\hline 1 & $\begin{array}{l}\text { Accurate Emotion } \\
\text { Detection }\end{array}$ & The model that can detect the emotions most accurately is required \\
\hline 2 & $\begin{array}{l}\text { Accurate Sentiment } \\
\text { Analysis }\end{array}$ & The model that has the most accurate sentiment analysis is required \\
\hline 3 & Emotion \\
Classification & $\begin{array}{l}\text { Casual Style Writing } \\
\text { required }\end{array}$ & $\begin{array}{l}\text { The model that corrects the text style to detect the emotions properly is } \\
\text { required }\end{array}$ \\
\hline 4 & Fuzzy Boundaries & $\begin{array}{l}\text { The model that covers the fuzzy boundaries and has a solution for them is } \\
\text { required }\end{array}$ \\
\hline 6 & Efficiency & $\begin{array}{l}\text { A model that is efficient in detecting emotions and sentiment analysis is } \\
\text { required }\end{array}$ \\
\hline 7 & Sentence Structure & The model that considers the sentence structures and fixes them is required \\
\hline 8 & Grammatical Mistakes & The model that considers grammatical mistakes and fixes them is required \\
\hline 9 & Emotion Variety & The model that detects the most amount of emotions is required \\
\hline 10 & Multiple Emotions & A model that can detect multiple emotions in text correctly is required \\
\hline
\end{tabular}

LGU Research Journal of Computer Science \& Information Technology 5(2) LGURJCSIT 
Table 2: Table of Comparative Analysis of Models based on Characteristics, Technologies, and Limitations

\begin{tabular}{|c|c|c|c|c|}
\hline Ref. & Proposed Model & Characteristic & Used Technologies & Limitations \\
\hline$[1]$ & $\begin{array}{l}\text { SENN (Semantic- } \\
\text { Emotion Neural } \\
\text { Network) }\end{array}$ & $\begin{array}{l}\text { - BiLSTM, CNN } \\
\text { - Accuracy }\end{array}$ & $\begin{array}{c}\text { Ubuntu } 17.10 \text {, Python } 3.5 \text {, } \\
\text { Scikit-learn }\end{array}$ & $\begin{array}{c}\text { - Performance } \\
\text { - Limited Emotions }\end{array}$ \\
\hline$[2]$ & BiLSTM-LM & $\begin{array}{l}\text { - Encode the vocabulary } \\
\text { - Represents text as the } \\
\text { word feature } \\
\text { representation. }\end{array}$ & $\begin{array}{c}\text { 64GB DDR4 2133MHz, } \\
\text { Linux Ubuntu 14.0, } \\
\text { Anaconda3, Python 3.5, } \\
\text { TensorFlow } 10.0\end{array}$ & $\begin{array}{l}\text { - Dictionary length } \\
\text { - Limited Emotions }\end{array}$ \\
\hline$[3]$ & $\begin{array}{l}\text { Textual and Emojis } \\
\text { Classifications by } \\
\text { Fuzzy Logic }\end{array}$ & $\begin{array}{c}\text { - FCS, TFCL, ECFL } \\
\text { - Text is important than } \\
\text { emojis }\end{array}$ & $\begin{array}{c}\text { Flask, Twitter API, Python } \\
\text { Text Blob Library, Plotly } \\
\text { Library }\end{array}$ & $\begin{array}{l}\text { - Models not united } \\
\text { - Number of emotions } \\
\text { and Reliability }\end{array}$ \\
\hline$[4]$ & $\begin{array}{c}\text { HNN (Hybrid } \\
\text { Neural Networks) }\end{array}$ & $\begin{array}{l}\text { - Pre-trained LSM } \\
\text { - Fine-tuned Hybrid } \\
\text { Model }\end{array}$ & $\begin{array}{c}\text { SWAT, ET, ETM, MSTM, } \\
\text { SLTM, ATM }\end{array}$ & $\begin{array}{c}\text { - Instability } \\
\text { - Limited Emotions }\end{array}$ \\
\hline$[5]$ & $\begin{array}{l}\text { Skip-Thought } \\
\text { Vectors for Emotion } \\
\text { Detection }\end{array}$ & $\begin{array}{l}\text { - Pre-trained model and } \\
\text { word vectors } \\
\text { - Lookup table layer }\end{array}$ & $\begin{array}{l}\text { Python, cPickle, NumPy, } \\
\text { Theano }\end{array}$ & $\begin{array}{c}\text { - Accuracy } \\
\text { - Need a large-scale } \\
\text { dataset }\end{array}$ \\
\hline$[6]$ & $\begin{array}{l}\text { Dimensional model, } \\
\text { Soft classification } \\
\text { approach \& } \\
\text { Supervised learning } \\
\text { system }\end{array}$ & $\begin{array}{l}\text { - Evaluates supervised } \\
\text { learning system }\end{array}$ & $\begin{array}{c}\text { Ubuntu 14.0, Anaconda3, } \\
\text { Python 3.5, TensorFlow } \\
10.0\end{array}$ & $\begin{array}{l}\text { - No consensus on the } \\
\text { basic set of emotions }\end{array}$ \\
\hline$[7]$ & $\begin{array}{l}\text { Unifying WordNet- } \\
\text { Affect and } \\
\text { EmoSenticNet }\end{array}$ & $\begin{array}{l}\text { - Unified model using } \\
\text { WordNet-Affect and } \\
\text { EmoSenticNet } \\
\text { - Accuracy }\end{array}$ & $\begin{array}{c}\text { Python, Twitter API, } \\
\text { Natural Language Toolkit }\end{array}$ & $\begin{array}{l}\text { - Small dataset } \\
\text { - Ambiguity }\end{array}$ \\
\hline$[8]$ & $\begin{array}{l}\text { Character-Aware } \\
\text { Convolutional } \\
\text { Recurrent Networks } \\
\text { with Self-Attention }\end{array}$ & $\begin{array}{c}\text { - Pre-trained word } \\
\text { embedding } \\
\text { - Vector representations of } \\
\text { emotions }\end{array}$ & $\begin{array}{l}\text { Vector representation and } \\
\text { attention mechanisms }\end{array}$ & $\begin{array}{c}\text { - Cannot detect } \\
\text { multiple emotions from } \\
\text { a text }\end{array}$ \\
\hline$[9]$ & $\begin{array}{l}\text { Multi-label } \\
\text { Learning Approach } \\
\text { for Emotion } \\
\text { Detection }\end{array}$ & $\begin{array}{l}\text { - Multiple Correlations } \\
\text { - Accuracy }\end{array}$ & $\begin{array}{l}\text { Python, Twitter API, } \\
\text { LangDetect, NLTK }\end{array}$ & $\begin{array}{c}\text { - Small dataset } \\
\text { - Limited Emotions }\end{array}$ \\
\hline [10] & $\begin{array}{l}\text { Semantic-based } \\
\text { model and VSM }\end{array}$ & $\begin{array}{l}\text { - Determine similarities } \\
\text { between short texts } \\
\text { - Uses the feature vector } \\
\text { cosine }\end{array}$ & $\begin{array}{c}\text { Anaconda3, Python } 3.5, \\
\text { NLTK }\end{array}$ & $\begin{array}{l}\text { - Path calculation } \\
\text { without the original } \\
\text { sense of the words }\end{array}$ \\
\hline [11] & $\begin{array}{l}\text { Emotion and } \\
\text { Sentiment Analysis } \\
\text { from Twitter Text }\end{array}$ & $\begin{array}{l}\text { - Recommendation } \\
\text { System }\end{array}$ & $\begin{array}{l}\text { NavigTweet, T-PICE, } \\
\text { Python, and NLTK }\end{array}$ & $\begin{array}{c}\text { - Simple Text } \\
\text { - Limited Emotions }\end{array}$ \\
\hline [12] & $\begin{array}{c}\text { Unigram and } \\
\text { Unigram with POS }\end{array}$ & $\begin{array}{l}\text { - Feature Extraction } \\
\text { - Naive Bayes }\end{array}$ & $\begin{array}{c}\text { Natural Language Tool Kit, } \\
\text { Python, and unigram } \\
\text { method }\end{array}$ & $\begin{array}{c}\text { - Accuracy } \\
\text { - Limited Emotions }\end{array}$ \\
\hline [13] & $\begin{array}{c}\text { Text Emotion } \\
\text { Detection in Social } \\
\text { Networks using } \\
\text { Novel Ensemble } \\
\text { Classifier Based on } \\
\text { TPE }\end{array}$ & $\begin{array}{c}\text { - Efficient } \\
\text { - Multilayer Perceptron, k- } \\
\text { Nearest Neighbour, DT } \\
98.3 \%\end{array}$ & $\begin{array}{c}\text { Python, Scikit-Learn, } \\
\text { NLTK }\end{array}$ & $\begin{array}{c}\text { - Performance } \\
\text { - Limited Emotions }\end{array}$ \\
\hline
\end{tabular}

LGU Research Journal of Computer Science \& Information Technology 5(2) LGURJCSIT 


\begin{tabular}{|c|c|c|c|c|}
\hline [14] & $\begin{array}{l}\text { Topic-enhanced } \\
\text { Capsule Network }\end{array}$ & $\begin{array}{l}\text { - Variational autoencoder } \\
\text { - Capsule module for } \\
\text { multi-level emotions }\end{array}$ & $\begin{array}{c}\text { Contextualized language } \\
\text { model BERT }\end{array}$ & $\begin{array}{l}\text { - Limited Emotions } \\
\text { - Topic modeling }\end{array}$ \\
\hline [15] & EmoLabel & $\begin{array}{l}\text { - Semi-Automatic } \\
\text { Methodology }\end{array}$ & Python and Scikit-Learn & - Limited Emotions \\
\hline$[16]$ & $\begin{array}{l}\text { Naive Bayes } \\
\text { Classifier }\end{array}$ & $\begin{array}{l}\text { - Particle swarm } \\
\text { optimization } \\
\text { - } 66.54 \% \text { accuracy }\end{array}$ & $\begin{array}{l}\text { Web 2.0-based technology } \\
\text { and Python }\end{array}$ & $\begin{array}{l}\text { - No detection while } \\
\text { typing } \\
\text { - Limited Emotions }\end{array}$ \\
\hline [17] & $\begin{array}{l}\text { Text-Based Emotion } \\
\text { Recognition in } \\
\text { Decision Support }\end{array}$ & $\begin{array}{l}\text { - Decision Support Model } \\
\text { - Performance } 58.1 \%\end{array}$ & $\begin{array}{c}\text { Sent2aff ect and lexicon- } \\
\text { based methods, NLTK, and } \\
\text { BiLSTM. }\end{array}$ & - Limited Emotions \\
\hline [18] & $\begin{array}{l}\text { Neural Network and } \\
\text { Feature Extraction } \\
\text { Model }\end{array}$ & $\begin{array}{l}\text { - Classification Accuracy } \\
\text { - NLP and } 91 \% \text { accuracy }\end{array}$ & $\begin{array}{l}\text { Python, TF-IDF, LSA, and } \\
\text { LDA approaches }\end{array}$ & $\begin{array}{l}\text { - Large Dimensions } \\
\text { - Performance }\end{array}$ \\
\hline [19] & $\begin{array}{l}\text { Lexicon Generation } \\
\quad \text { for Emotion } \\
\text { Detection from Text }\end{array}$ & $\begin{array}{l}\text { - Unigram Emotional } \\
\text { Model } \\
61.7 \%\end{array}$ & $\begin{array}{l}\text { NLTK, Fuzzy clustering, } \\
\text { PMI, and LDA techniques }\end{array}$ & $\begin{array}{l}\text { - No Multi-Word } \\
\text { Lexicon } \\
\text { - Limited Emotions }\end{array}$ \\
\hline [20] & $\begin{array}{c}\text { Feature-level Fusion } \\
\text { Model }\end{array}$ & $\begin{array}{l}\text { - Self-attention } \\
\text { mechanism }\end{array}$ & $\begin{array}{c}\text { Fusion platforms like } \\
\text { multidimensional attention } \\
\text { to test }\end{array}$ & $\begin{array}{l}\text { - Degradation of the } \\
\text { system }\end{array}$ \\
\hline [21] & $\begin{array}{l}\text { Emotion Detection } \\
\text { in Text using Nested } \\
\text { LSTM }\end{array}$ & $\begin{array}{l}\text { - Machine Learning } \\
\text { - 98.86\% accuracy }\end{array}$ & $\begin{array}{l}\text { LSTM, Nested LSTM, } \\
\text { Python, and NLTK }\end{array}$ & - Limited Emotions \\
\hline [22] & $\begin{array}{l}\text { Hybrid Model to } \\
\text { Detect Depression } \\
\text { from Reddit text }\end{array}$ & $\begin{array}{l}\text { - Feature extraction model } \\
\text { - Text classification model }\end{array}$ & $\begin{array}{l}\text { BiLSTM, word embedding, } \\
\text { and Trained dataset of Deep } \\
\text { Learning }\end{array}$ & $\begin{array}{c}\text { - Performance } \\
\text { - Limited Emotions }\end{array}$ \\
\hline [23] & $\begin{array}{l}\text { Hybrid Model for } \\
\text { Emotion Detection }\end{array}$ & $\begin{array}{l}\text { - Hybrid Model } \\
\text { - Semantic Similarities } \\
\text { and } 0.766 \text { Precision }\end{array}$ & $\begin{array}{l}\text { VSM, LSA, PLSA, NMF, } \\
\text { Python, and NLTK }\end{array}$ & - Limited Emotions \\
\hline [24] & $\begin{array}{c}\text { Emotion Extraction } \\
\text { Model }\end{array}$ & $\begin{array}{l}\text { - Text pre-processing } \\
\text { - Keyword extraction \& } \\
\text { analysis and } 65 \% \\
\text { accuracy }\end{array}$ & $\begin{array}{l}\text { Predefined emotional } \\
\text { keyword database }\end{array}$ & $\begin{array}{c}\text { - Accuracy } \\
\text { - Limited Emotions }\end{array}$ \\
\hline$[25]$ & EmoTxt & $\begin{array}{l}\text { - Open-source toolkit } \\
\text { - Gold standard dataset }\end{array}$ & SVM, Python, and NLTK & $\begin{array}{l}\text { - Varying Sources not } \\
\text { used } \\
\text { - Limited Emotions }\end{array}$ \\
\hline
\end{tabular}

reliable emotion detection which could, later on, be introduced into real-world systems. The main objective of this review paper is to analyze models and determine which model ends up being the most reliable and accurate one for practical real-life applications. While accuracy and performance are one of the main factors that determine which model should be selected, there are other factors as well that can heavily impact the final verdict. Some models discussed in
Section III did not even take complex sentence structures and the existence of multiple emotions in a sentence into consideration. Some models had a bad performance which is not practical for real-world systems. Some models did consider that but suffered from bad accuracy. One of the other drawbacks we found in the proposed studies is casual style writing, complex sentence structures, and grammar mistakes which need to be addressed for perfect 
emotion detection from the text. Any new model introduced needs to consider all these challenges and without solving these challenges their models end up being un-applicable for realworld use. Another huge drawback we found in every model we studied was that every model lacked a variety of emotions, the proposed models relied on either the old version of Ekman's Emotions (happy, anger, sad, fear, disgust, and surprised) or Plutchik's Emotions (joy, trust, fear, surprise, sadness, anticipation, anger, and disgust) which are not enough for practical real-world applications. For an application to be applicable in a real-world scenario, the model has to take into consideration that humans have a wide variety of emotions and sub-emotions.

\subsection{Recommendations}

After a considerable amount of research and discussions, our contribution is that we determined the challenges discussed in Section II and compared them to the models discussed in Section III. The models had to be efficient as well as take other challenges such as casual writing styles, fuzzy boundaries, sentence structures, grammatical mistakes, and multiple emotions into consideration. We had to make a compromise on emotion variety since all the models discussed didn't use a large variety of emotions and relied on old versions of Ekman's Classification of Emotions or Plutchik's Classification of Emotions. The models that did well against the challenges we did take into consideration were Parzen Tree Estimator based Text Emotion Detection in Social Networks using Novel Ensemble Classifier [13], Neural Network and Feature Extraction Model [18], and Emotion Detection in Text using Long Short-Term and Nested Long Short-Term Memory [21]. The first two models did have very high accuracy but they are not recommended since the first one does not have good efficiency and the second one had issues with large dimensions which lead to the performance issues. The third model had the best performance of the overall discussed models as well as had a good performance, the only drawback was the variety of emotions used which as we talked about before wasn't taken into consideration to judge the models since all models used classifications of emotions that had a small variety of emotions.

\section{CONCLUSION}

This paper elaborates the mechanisms of different emotion detection models by the processes of Keyword Based Techniques, Learning-Based Techniques, Lexical Affinity Techniques, and lastly the Hybrid Method. While not all the challenges of the existing systems are overcome in newly proposed models, they do however propose models with increased efficiency and accuracy. Based on our observation and research, we discussed the existing models we studied and then proposed our recommendations. While we do think that the model recommended can be used in the practical application based on accuracy and efficiency as well as the challenges they took into consideration, we still think future work needs to be done so that variety of emotions can be introduced into these models for a wide range of real-world implementations.

\section{REFERENCES}

[1] Batbaatar, E., Li, M. and Ryu, K.H., Semantic-emotion neural network for emotion recognition from text, IEEE Access, 7, pp.111866-111878. 2019.

[2] Zheng, J., A Novel Computer-Aided Emotion Recognition of Text Method Based on WordEmbedding and BiLSTM. In 2019 International Conference on Artificial Intelligence and Advanced Manufacturing (AIAM), (pp. 176-180). IEEE. 2019.

[3] Tashtoush, Y.M. and Orabi, D.A.A.A., Tweets Emotion Prediction by Using Fuzzy Logic System. In 2019 Sixth International Conference on Social Networks Analysis, Management and Security (SNAMS), pp. 83-90, 2019.

[4] Li, X., Pang, J., Mo, B. and Rao, Y., Hybrid neural networks for social emotion detection over short text. In 2016 International joint conference on neural networks (IJCNN), pp. 537-544. 2016.

[5] Hassan, M., Alam, M.S.B. and Ahsan, T., Emotion Detection from Text Using Skip-thought Vectors. In 2018 International Conference on Innovations in Science, Engineering and Technology (ICISET), pp. 501-506, 2018. 
[6] Hasan, M., Rundensteiner, E. and Agu, E., Automatic emotion detection in text streams by analyzing twitter data. International Journal of Data Science and Analytics, 7(1), pp.35-51, 2019.

[7] Shah, F.M., Reyadh, A.S., Shaafi, A.I., Ahmed, S. and Sithil, F.T., Emotion detection from tweets using AIT-2018 dataset. In 20195 th International Conference on Advances in Electrical Engineering (ICAEE), pp. 575-580, 2019.

[8] Huang, J., Xiang, C., Yuan, S., Yuan, D. and Huang, X., Character-aware convolutional recurrent networks with self-attention for emotion detection on Twitter. In 2019 International Joint Conference on Neural Networks, pp. 1$8,2019$.

[9] Zhang, X., Li, W., Ying, H., Li, F., Tang, $\mathrm{S}$. and Lu, S., Emotion Detection in Online Social Networks: A Multilabel Learning Approach. IEEE Internet of Things Journal, 7(9), pp.8133-8143, 2020 .

[10] Mozafari, F. and Tahayori, H., Emotion detection by using similarity techniques. In 2019 7th Iranian Joint Congress on Fuzzy and Intelligent Systems, pp. 1-5, 2019.

[11] Sailunaz, K. and Alhajj, R., Emotion and sentiment analysis from Twitter text. Journal of Computational Science, 36, p.101003, 2019.

[12] Sharupa, N.A., Rahman, M., Alvi, N., Raihan, M., Islam, A. and Raihan, T., Emotion Detection of Twitter Post using Multinomial Naive Bayes. In 2020 11th International Conference on Computing, Communication and Networking Technologies, pp. 1-6, 2020.

[13] Ghanbari-Adivi, F. and Mosleh, M., Text emotion detection in social networks using a novel ensemble classifier based on Parzen Tree Estimator (TPE). Neural Computing and Applications, 31(12), pp.8971-8983, 2019.
[14] Fei, H., Ji, D., Zhang, Y. and Ren, Y., Topic-enhanced capsule network for multi-label emotion classification. IEEE/ACM Transactions on Audio, Speech, and Language Processing, 28, pp.1839-1848, 2020.

[15] Canales, L., Daelemans, W., Boldrini, E. and Martínez-Barco, P., EmoLabel: Semi-Automatic Methodology for Emotion Annotation of Social Media Text. IEEE Transactions on Affective Computing, 2019.

[16] Junianto, E. and Rachman, R., Implementation of Text Mining Model to Emotions Detection on Social Media Comments Using Particle Swarm Optimization and Naive Bayes Classifier. In 20197 th International Conference on Cyber and IT Service Management, Vol. 7, pp. 1-6, 2019.

[17] Kratzwald, B., Ilic, S., Kraus, M., Feuerriegel, S. and Prendinger, H., Deep learning for affective computing: Textbased emotion recognition in decision support. Decision Support Systems, 115, pp.24-35, 2018.

[18] Dzisevic, R. and Šešok, D., 2019, April. Text Classification Using Different Feature Extraction Approaches. In 2019 Open Conference of Electrical, Electronic and Information Sciences, pp. $1-4,2019$.

[19] Bandhakavi, A., Wiratunga, N., Massie, S. and Padmanabhan, D., Lexicon generation for emotion detection from text. IEEE intelligent systems, 32(1), pp.102-108, 2017.

[20] Hazarika, D., Gorantla, S., Poria, S. and Zimmermann, R., 2018, April. Selfattentive feature-level fusion for multimodal emotion detection. In 2018 IEEE Conference on Multimedia Information Processing and Retrieval, pp. 196-201, 2018.

[21] Haryadi, D. and Kusuma, G.P., Emotion Detection in Text using Nested Long Short-Term Memory. 11480 (IJACSA) International Journal of Advanced 
Computer Science and Applications, 10(6), 2019.

[22] Shah, F.M., Ahmed, F., Joy, S.K.S., Ahmed, S., Sadek, S., Shil, R. and Kabir, M.H., Early Depression Detection from Social Network Using Deep Learning Techniques. In 2020 IEEE Region 10 Symposium, pp. 823-826, 2020.

[23] Fathy, S., El-Haggar, N. and Haggag, M.H., A hybrid model for emotion detection from text. International Journal of Information Retrieval Research (IJIRR), 7(1), pp.32-48, 2017.

[24] Seal, D., Roy, U.K. and Basak, R., 2020. Sentence-level emotion detection from text based on semantic rules. In Information and Communication Technology for Sustainable Development, pp. 423-430, 2020.

[25] Calefato, F., Lanubile, F. and Novielli, N., Emotxt: a toolkit for emotion recognition from text. In 2017 seventh international conference on Affective Computing and Intelligent Interaction Workshops and Demos, pp. 79-80, 2017.

[26] Ekman, P., Basic emotions. Handbook of cognition and emotion. Wiley, New York, pp.301-320, 1999.

[27] Plutchik, R., The emotions. University Press of America, 1991.

[28] Ashraf, M.U., Eassa, F.A., Albeshri, A.A. and Algarni, A., Performance and power efficient massive parallel computational model for HPC heterogeneous exascale systems. IEEE Access, 6, pp.23095-23107, 2018.

[29] Ashraf, M.U., Qayyum, R. and Ejaz, H., State-of-the-art, Challenges: Privacy Provisioning In Ttp Location Based Services Systems. International Journal of Advanced Research in Computer Science(IJARCS), 10(2), 2019.

[30] Fatima, F., Ali, S. and Ashraf, M.U., Risk Reduction Activities Identification in Software Component Integration for Component Based Software
Development (CBSD). International Journal of Modern Education and Computer Science, 9(4), p. 19, 2017.

[31] Siddiqui, N., Yousaf, F., Murtaza, F., Ehatisham-ul-Haq, M., Ashraf, M.U., Alghamdi, A.M. and Alfakeeh, A.S., A highly nonlinear substitution-box (Sbox) design using action of modular group on a projective line over a finite field. Plos one, 15(11), p.e0241890, 2020.

[32] Javed, R., Anwar, S., Bibi, K., Ashraf, M.U. and Siddique, S., Prediction and monitoring agents using weblogs for improved disaster recovery in cloud. Int. J. Inf. Technol. Comput. Sci.(IJITCS), 11(4), pp.9-17, 2019.

[33] Ashraf, M.U., Arif, S., Basit, A. and Khan, M.S., Provisioning quality of service for multimedia applications in cloud computing. Int. J. Inf. Technol. Comput. Sci.(IJITCS), 10(5), pp.40-47, 2018.

[34] Tariq, S., Ahmad, N., Ashraf, M.U., Alghamdi, A.M. and Alfakeeh, A.S., Measuring the Impact of Scope Changes on Project Plan Using EVM. IEEE Access, 8, pp.154589-154613, 2020.

[35] Alsubhi, K., Ashraf, M.U. and Ilyas, I., HBLP: A Privacy Protection Framework for TIP Attributes in NTTP-Based LBS Systems. IEEE Access, 8, pp.6771867734, 2020.

[36] Alrahhal, M.S., Ashraf, M.U., Abesen, A. and Arif, S., AES-route server model for location based services in road networks. International Journal of Advanced Computer Science And Applications, 8(8), pp.361-368, 2017.

[37] Riaz, S., Ashraf, M.U. and Siddiq, A., A Comparative Study of Big Data Tools and Deployment PIatforms. In 2020 International Conference on Engineering and Emerging Technologies, pp. 1-6, 2020.

[38] Alsubhi, K., Imtiaz, Z., Raana, A., Ashraf, M.U. and Hayat, B., MEACC: an 
energy-efficient framework for smart devices using cloud computing systems. Frontiers of Information Technology \& Electronic Engineering, 21(6), pp.917$930,2020$.

[39] Abid, U. and Ashraf, M.U., February. A Critical Survey On Privacy Prevelling In Collaborative Filtring Recomender System: Challenges, State-Of-The-Art Methods And Future Directions. In 2020 International Conference on Engineering and Emerging Technologies (ICEET), pp. 1-7, 2020.

[40] Manzoor, A., Ahmad, W., Ehatisham-ulHaq, M., Hannan, A., Khan, M.A., Ashraf, M.U., Alghamdi, A.M. and Alfakeeh, A.S., Inferring Emotion Tags from Object Images Using Convolutional Neural Network. Applied Sciences, 10(15), p.5333, 2020.

[41] Alsubhi, K., Alsolami, F., Algarni, A., Albassam, E., Khemakhem, M., Eassa, F., Jambi, K. and Ashraf, M.U., A Tool for Translating sequential source code to parallel code written in $\mathrm{C}++$ and OpenACC. In 2019 IEEE/ACS 16th International Conference on Computer Systems and Applications (AICCSA), pp. 1-8, 2019. 